\title{
A guidewire-assisted biopsy technique to assist advancement through a biliary stricture to perform selective mapping biopsy
}

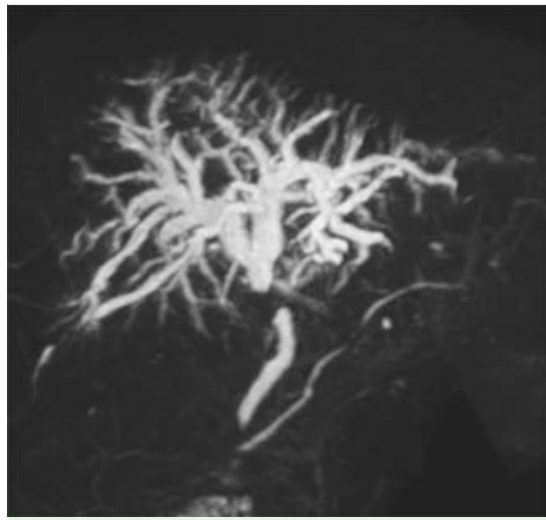

Fig. 1 Magnetic resonance cholangiography image in a 69-year-old woman with obstructive jaundice showing a hilar biliary stricture.

An endoscopic transpapillary mapping biopsy is useful for evaluating intraepithelial tumor spread in bile duct cancers $[1,2]$; however, it is occasionally technically difficult to advance the biopsy forceps through a biliary stricture. Here, we describe the case of a patient with a hilar biliary stricture who underwent a selective mapping biopsy. The biopsy technique described is suitable where the bile duct is inaccessible when introducing conventional biopsy forceps.

A 69-year-old woman presented with obstructive jaundice. Magnetic resonance cholangiography indicated a hilar biliary stricture ( Fig. 1). We attempted a transpapillary intraductal biopsy of the biliary stricture and a mapping biopsy using conventional biopsy forceps to confirm the definitive diagnosis and look for proximal intraepithelial tumor spread; however, the forceps could not be passed through the stricture. Therefore, we attempted a guidewire-assisted intraductal biopsy.

First, a 0.025-inch guidewire (VisiGlide $2^{\mathrm{TM}}$; Olympus Medical Systems, Tokyo, Japan) was placed at the left intrahepatic bile duct. The guidewire was gripped by the biopsy forceps (Radial Jaw ${ }^{\mathrm{TM}} 4 \mathrm{P}$; Boston Scientific Japan, Tokyo, Japan) outside the papilla ( Fig. 2). Thereafter, the biopsy forceps, which were still securely gripping the guidewire, were slid into the bile duct and advanced through the hilar biliary stricture under fluoroscopic guidance
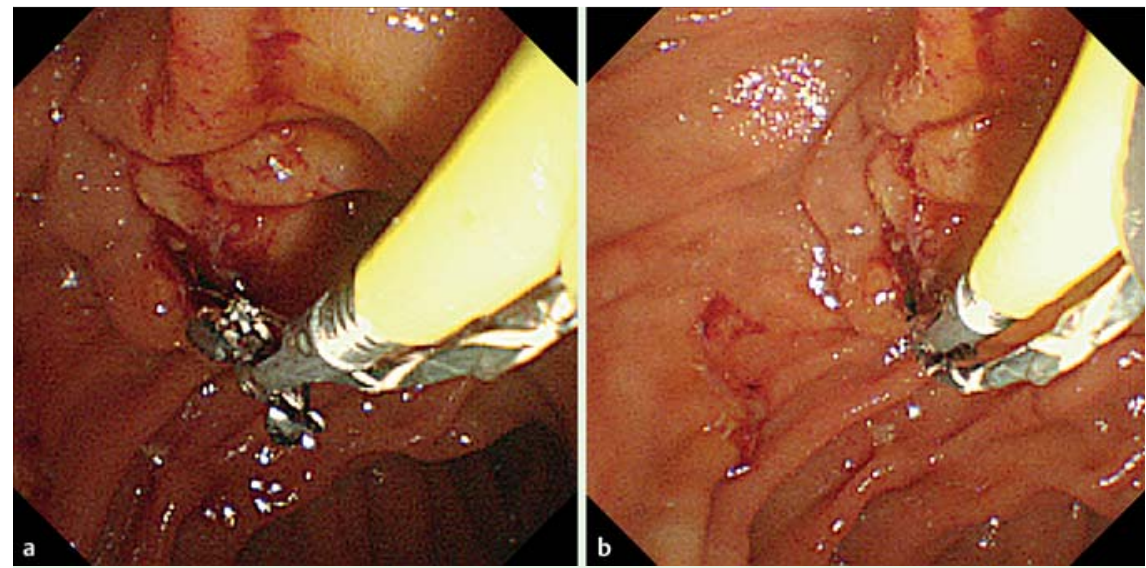

Fig. 2 Endoscopic image showing the jaw configuration biopsy forceps: a open and positioned just outside the papilla; $\mathbf{b}$ gripping the guidewire outside the papilla.

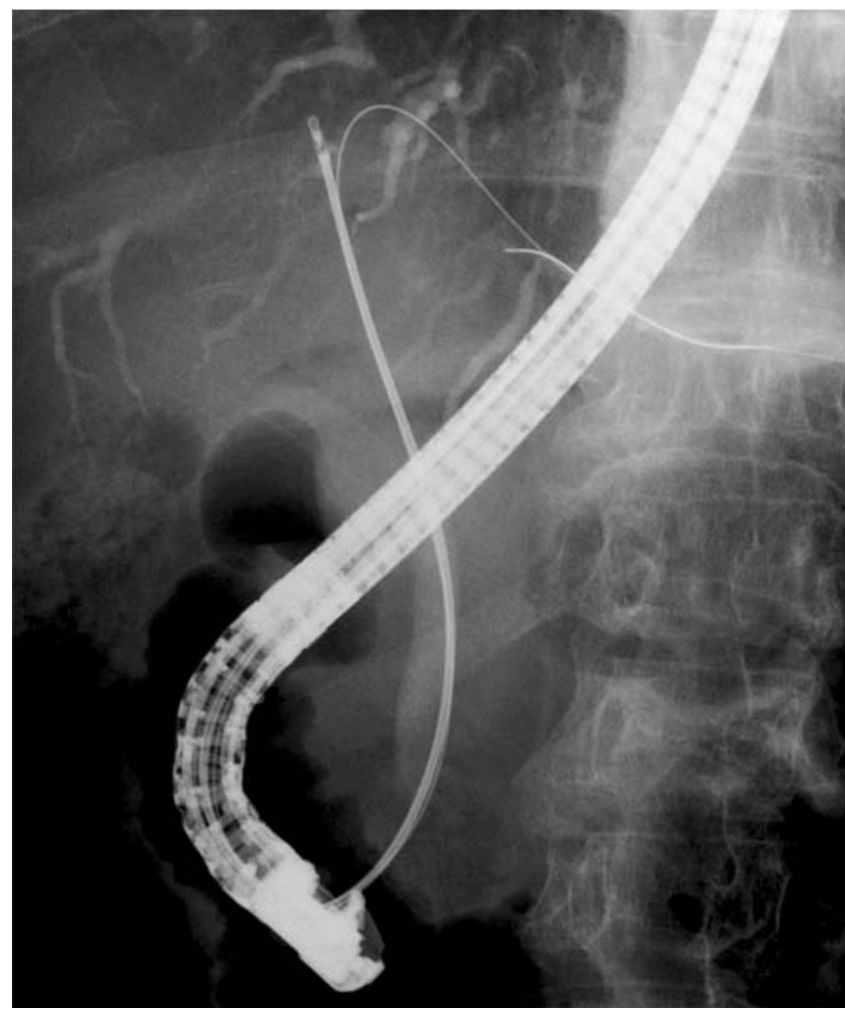

Fig. 3 Fluoroscopic image showing the biopsy forceps gripping the guidewire, while being advanced through the biliary stricture.

( $\bullet$ Fig. 3, Video 1). Finally, a successful biopsy was performed from the B4 confluence without any complications occurring. Intraductal biopsy specimens of biliary strictures can be obtained under fluoroscopic guidance using conventional biopsy forceps, biliary introducers [3], or rope-

\section{Video 1}

Guidewire-assisted technique using jaw configuration biopsy forceps to access the proximal bile duct through a hilar biliary stricture in order to evaluate intraepithelial tumor spread. 
way-type biopsy forceps [4]. Biopsy forceps are, however, occasionally difficult to advance through a biliary stricture. The use of miniforceps with a cut biliary dilator was recently reported [2]. Li et al. [5] introduced a guidewire-assisted technique to access the bile duct. To our knowledge, this is the first report of a guidewire-assisted biopsy beyond a biliary stricture. This biopsy technique not only helps with accessing the bile duct but also aids advancement of the forceps through the biliary stricture.

Endoscopy_UCTN_Code_TTT_1AR_2AD

\section{Competing interests: None}

Hiroshi Kawakami ${ }^{1}$, Masaki Kuwatani², Yoko Abe', Shuhei Kawahata', Kazumichi Kawakubo ${ }^{3}$, Kimitoshi Kubo $^{1}$, Naoya Sakamoto ${ }^{3}$

${ }^{1}$ Department of Gastroenterology and Hepatology, Hokkaido University Hospital, Sapporo, Japan

2 Division of Endoscopy, Hokkaido University Hospital, Sapporo, Japan

${ }^{3}$ Department of Gastroenterology and Hepatology, Hokkaido University Graduate School of Medicine, Sapporo, Japan

\section{References}

1 Kawakami H, Kuwatani M, Etoh $K$ et al. Endoscopic retrograde cholangiography versus peroral cholangioscopy to evaluate intraepithelial tumor spread in biliary cancer. Endoscopy 2009; 41: 959-964

2 Hijioka S, Hara K, Mizuno $N$ et al. A novel technique for endoscopic transpapillary "mapping biopsy specimens" of superficial intraductal spread of bile duct carcinoma (with videos). Gastrointest Endosc 2014; 79: $1020-1025$

3 Howell DA, Parsons WG, Jones MA et al. Complete tissue sampling of biliary strictures at
ERCP using a new device. Gastrointest Endosc 1996; 43: 498-502

4 Tamada K, Higashizawa T, Tomiyama T et al. Rope-way bile duct forceps with a slide slit for a guidewire. Gastrointest Endosc 2001; 53: 89-92

5 Li QY, Chen KJ, Zheng SS. Guide wire-assisted technique to access the bile duct with biopsy forceps for repositioning and removal of metal stents. Endoscopy 2013; 45: E273E274

\section{Bibliography}

DOI http://dx.doi.org/

10.1055/s-0034-1391827

Endoscopy 2015; 47: E217-E218

(c) Georg Thieme Verlag KG

Stuttgart · New York

ISSN 0013-726X

\section{Corresponding author}

Hiroshi Kawakami, MD, PhD

Department of Gastroenterology and Hepatology Hokkaido University Hospital

Kita 14, Nishi 5, Kita-ku

Sapporo 060-8648

Japan

Fax: +81-11-7067867

hiropon@med.hokudai.ac.jp 